\title{
Evaluation of the Macula, Retinal Nerve Fiber Layer and Choroid Thickness in Women Using Oral Contraceptive Pills
}

\author{
MAGDA M.M. SAMY, M.D.; MOUAMEN M. MOSTAFA, M.D; NASHWA M. EZZAT, M.D. and \\ EMAN M.G. EL-SAID, M.Sc.
}

The Department of Ophthalmology, Faculty of Medicine, Ain Shams University

\begin{abstract}
Background: Oral Contraceptive Pills (OCP) have some risks and side effects with regard to several organs, one of which is the eye. Estrogen \& progesterone receptors have been shown to be present in various eye structures, such as the choroid, retina, conjunctiva, cornea, Meibomian glands, and lens.
\end{abstract}

Aim of Study: The purpose of this study is to assess the macula, Retinal Nerve Fiber Layer (RNFL), and choroid thickness, in addition to evaluation of macular Ganglion Cell Complex (GCC) by using spectral-domain OCT system (3D OCT-2000 FA; Topcon Crop., Tokyo, Japan) in 20 women using Oral Contraceptive Pills (OCP) for more than one year and to compare them with 20 healthy reproductive age women volunteers.

Patients and Methods: This is cross sectional study included 20 women using oral contraceptive pills in comparison to 20 normal females between April 2018 till July 2018 at Giza memorial Institute of Ophthalmic Research. Optical Coherence Tomography (OCT) is used to evaluate the posterior ocular segments, and measurements have been taken in the follicular phase (day 3 ) of the cycle in all women.

Results: The mean age of the OCP group was $31.7 \pm 3.76$ years and the mean age of the control group was $31.3 \pm 5.031$ years. Both groups are homogenous in terms of age $(p=0.7773)$. Most of the macular layer thickness and the foveal center are significantly thinner in OCP group. The macular volume is thinner in OCP study group. Retinal nerve fiber layers are thicker in OCP study group a part from Superior RNFL which is thinner in OCP study group, while temporal RNFL zone is with no significant changes. Choroid thickness values, is thicker in OCP study group. Ganglion cell complex is with no significant difference.

Conclusion: We found that women who had received combined oral contraceptive pills more than one year have changes in posterior ocular segment.

Key Words: Macula - Retinal nerve fiber layer - Choroid thickness - Oral contraceptive pills.

Correspondence to: Dr. Eman M.G. El-Said, E-Mail: emanmagdy2@gmail.com

\section{Introduction}

ORAL Contraceptive Pills (OCP) have some risks and side effects with regard to several organs, one of which is the eye. Estrogen \& progesterone receptors have been shown to be present in various eye structures, such as the choroid, retina, conjunctiva, cornea, Meibomian glands, and lens [1]

Egypt demographic and health survey (EDHS), 2005 provided that $57.5 \%$ of the married women in Egypt were using contraception. The most widely used methods are Intra Uterine Device (IUD), Oral Contraceptive Pill (OCP), and injection (respectively, $33.1 \%, 10.8 \% 7.7 \%$ ) [2].

Over 100 million women around the world use hormonal contraceptive methods, whereas 93 million of them use combined oral contraceptive pills (COCs) [3].

The incidence of ocular side effects of oral contraception can be calculated as 1:230,000 in the United States, Canada, Australia, 1:180,000 in the German Federal Republic, and 1:14,000 in Great Britain [4] .

Combined oral contraceptive pills (COCs) are widely indicted for contraception, menorrhagia, endometriosis, acne and hirsutism, fibroid uterus and premenstrual syndrome. The incidence of venous thromboembolic disease in healthy female of reproductive age receiving COCs is increased from 4-5/10000 women to $9-10 / 10000$ [5].

Ocular complications or side effects reported in connection with oral contraceptives include dry eye symptoms, corneal disturbances, lens opacities, retinal vascular disorders, and proptosis, in addition, retinal edema, glaucoma, and hemorrhagic retinopathy have also been reported [6]. 
Severe neuro-ophthalmologic complications involve the 6 th cranial nerve paralysis, parietal syndrome, hemianopsia, papillary edema and retrobulbar neuritis. Central retinal artery or vein occlusion, intraocular hemorrhages, aneurysms, macular or papillary edema and acute ischemic optic neuropathy represent the vascular complications of contraceptive pills [7]

Glaucoma is a serious condition that can develop from the use of oral contraceptives. The disease is characterized by damage to nerve tissue leading to a gradual loss of peripheral vision. It's not entirely clear why contraceptive use would possibly correlate to developing glaucoma, but optic nerve cells contain estrogen receptors that play a role in protecting the eyes from age-related decline. Research suggests that contraceptives could interfere in that process by lowering estrogen levels [8].

The biological actions of progestin are regulated by its receptors that belong in a superfamily of almost fifty ligand-activated nuclear transcription factors. This steroid receptor family includes the progestein, estrogen, androgen, glucocorticoid and mineralocorticoid receptors [9].

Sex hormones can affect the retina and choroid, so that OCP use could lead to result in alterations to OCT findings. Additionally, a correlation between sex hormones and disorders that may cause loss of vision has been described [10]

There could be a relationship between eyesight and estrogen, which have a protective effect on the eye. Therefore, it makes sense that the pills, which lowers a woman's total exposure to estrogen over a month, could affect her eyes. Birth control pills suppress cyclical spikes in women's estrogen levels, and over time, that dampening may contribute to retinal damage [11].

Estrogens seem to improve the symptoms of keratoconjunctivitis sicca and exhibit protective effect against glaucoma, cataractogenesis and degradation of corneal collagen. Moreover, the antioxidant and neuroprotective action of estrogens indicates their possible therapeutic use in neurodegenerative eye diseases, as well as AMD and diabetic retinopathy [12].

On the other hand, progesterone receptors are present on the arterial smooth-muscle walls, and that the role of progesterone is to counteract estrogen by reducing endothelial nitric oxide production $[13,14]$.
As a result of these mechanisms, flow-mediated vasodilatation can be antagonized when progesterone is added to the structure with estrogen [15] Thus, it may be that by the same mechanism, OCP may also have an effect on eye structure, as progestogenic activity of OCP dominates [16].

Optical Coherence Tomography (OCT), is a noncontact, noninvasive imaging technique that can reveal layers of the retina by looking at the interference patterns of reflected laser light [17].

Spectral-Domain Optical Coherence Tomography (SD-OCT) used for both quantitative and qualitative evaluation of the retina, and give us the potential to study various retinal layers more precisely in less time. It can delineate structural changes and fine lesions in the individual retinal layers

Several studies have tried to identify the ocular complications related to combined oral contraceptive pills, which observed that the use of OCP changes eye structures. Women using OCP for more than one year may have some eye problems

\section{Patients and Methods}

This is cross sectional study include 20 women using orals contraceptive pills in comparison to 20 normal females between April 2018 till July 2018 at Giza Memorial Institute of Ophthalmic Research.

Optical Coherence Tomography (OCT) is used to evaluate the posterior ocular segments, and measurements have been taken in the follicular phase (day 3 ) of the cycle in all women.

Participant females in our study group are receiving monophasic oral contraceptive pills $(0.03 \mathrm{mg}$ ethinylestradiol, $0.075 \mathrm{mg}$ gestodene) for more than one year.

All patients give an oral consent to participate in the study.

\section{Study inclusion criteria:}

- Female patients in the reproductive period.

- Age 18-45 years old.

- Healthy posterior segment appearance on fundus examination with slit lamp bio-microscopy.

- Receiving oral contraceptive pills more than 1 year prior to examination.

Study exclusion criteria:

- Age less than 18 or more than 45 years. 
- Pregnancy.

- Any associated systemic medical condition.

- Any associated ocular pathology.

- Ocular hypertension (Iop $>21 \mathrm{mmHg}$ with no diagnostic signs of glaucoma).

- Intake of any other medication.

- History of tobacco smoking.

\section{All patients underwent:}

Full medical history for having any chronic disease (e.g., Hypertension (HTN), Diabetes Mellitus (DM), thyroid disorders, thromboembolic disease, cardiovascular events, Cushing disease, positive malignancy, congenital adrenal hyperplasia, liver disease use steroidal hormone drugs.

Full ocular history taking for any chronic eye condition (e.g., glaucoma, cystoid macular edema, macular generation, optic atrophy, intraocular pressure $>21 \mathrm{mmHg}$, cataract, uveitis, etc.), previous ocular surgery (e.g., cataract, glaucoma, vitrectomy, etc.), past history of eye trauma.

\section{Ocular examination:}

- Best corrected visual acuity in snellen lines, and also calculated in logarithm of minimum angle of resolution ( $\log \mathrm{MAR})$.

- IOP measurement by Goldman applanation tonometry.

Posterior segment examination using the indirect ophthalmoscope and slit lamp bio microscopy with volk non-contact $+90 \mathrm{D}$ lens to assess the macula, optic nerve head and determine C/D ratio.

Examination technique of $\mathrm{ONH}$ and macular area by non-contact slit lamp 900 lens:

- Observe the size, shape, margins of the optic nerve head along with the cup-to-disc ratio.

- Evaluation of optic disc color, noting if it was pink or pale.

-Assessment of the macular area (macula is located 2.5-disc distance temporal to disc).

- Retinal Vessels assessment: AV ratio, AV crossing.

- Fundus background assessment.

Optical coherence tomography imaging:

- Spectral-domain OCT system (3D OCT-2000 FA; Topcon Corp., Tokyo, Japan) used to assess macula, RNFL and choroid thickness, in addition to evaluation of macular Ganglion Cell Complex (GCC). All OCT examinations were conducted by the same operator and using the same OCT instrument. The procedure was carried out under dim room lighting and without pupillary dilatation.

- The subject was seated with her chin in a chin rest and the machine properly aligned. The subject was then instructed to fixate with the eye being measured on the internal fixation target.

- Fast macular thickness map was used for macular evaluations, such as macular thickness and volume.

- Optic nerve head scan protocol calculates various parameters that describe the $\mathrm{ONH}$ and also generates a polar RNFL thickness map, which is the RNFL thickness measured along a circle $3.45 \mathrm{~mm}$ in diameter centered on the optic disc.

- Ganglion Cell Complex scan (GCC) is designed to automatically measure the inner retinal thickness, which includes the nerve fiber layer, ganglion cell layer, and the inner plexiform layer, collectively called the GCC.

- The choroidal thickness is measured manually perpendicularly from the outer edge of the hyperreflective RPE to the inner sclera (choroid-sclera junction) using the OCT software. The sub-foveal choroidal thickness was measured as the distance between the outer border of the RPE-Bruch's membrane complex and the chorio-scleral border under the fovea.

\section{Statistical analysis:}

Data were collected, revised, coded and entered to the statistical package for social science (IBM SPSS) Version 24. Probability ( $p$-value): $p$-value $<0.05$ was considered significant, $p$-value $<0.001$ was considered as highly significant, $p$-value $>0.05$ was considered insignificant.

\section{Results}

Table (1): The different age group of study participant cases. $\mathrm{OCP}$, oral contraceptive pills group and normal study group.

\begin{tabular}{lllllr}
\hline \multirow{2}{*}{ Age } & \multicolumn{2}{l}{ OCP group $(\mathrm{N}=20)$} & & \multicolumn{2}{l}{ Normal group $(\mathrm{N}=20)$} \\
\cline { 2 - 3 } \cline { 6 - 6 } & $\mathrm{N}$ & & $\mathrm{N}$ & $\%$ \\
\hline $18-29$ & 7 & 35 & & 9 & 45 \\
$30-39$ & 12 & 60 & & 9 & 45 \\
$40-50$ & 1 & 5 & 2 & 10 \\
\hline
\end{tabular}

This table shows the range of ages in participant in our study which declare that the most of OCP group participant aged ranging between 30-39 years old ( $3 \mathrm{rd}$ decade), followed by age ranging between 18-19 years old ( 2 nd decade). 
Table (2): Comparison between OCP group and normal group regarding macular thickness.

\begin{tabular}{|c|c|c|c|c|c|c|c|c|c|c|c|}
\hline & \multicolumn{4}{|c|}{ OCP group $(\mathrm{N}=40)$} & \multicolumn{4}{|c|}{ Normal group $(\mathrm{N}=40)$} & \multirow{2}{*}{ * } & \multirow{2}{*}{$\begin{array}{c}p- \\
\text { value }\end{array}$} & \multirow{2}{*}{ Sig } \\
\hline & Min. & Max. & Mean & SD & Min. & Max. & Mean & $\mathrm{SD}$ & & & \\
\hline Superior parafoveal macula & 258.00 & 317.00 & 292.05 & 17.25 & 230.00 & 331.00 & 304.38 & 22.26 & 2.77 & 2.77 & HS \\
\hline Temporal parafoveal macula & 236.00 & 294.00 & 269.88 & 14.28 & 251.00 & 315.00 & 294.60 & 10.66 & 8.78 & 8.78 & HS \\
\hline Inferior parafoveal macula & 237.00 & 311.00 & 282.40 & 17.80 & 266.00 & 320.00 & 300.52 & 10.84 & 5.50 & 5.50 & HS \\
\hline Nasal parafoveal macula & 254.00 & 321.00 & 287.68 & 18.56 & 270.00 & 326.00 & 309.38 & 11.56 & 6.28 & 6.28 & HS \\
\hline Superior perifoveal macula & 233.00 & 277.00 & 258.40 & 12.00 & 232.00 & 383.00 & 284.68 & 34.48 & 4.55 & 4.55 & HS \\
\hline Temporal perifoveal macula & 219.00 & 266.00 & 239.00 & 13.45 & 217.00 & 350.00 & 255.22 & 17.75 & 4.61 & 4.61 & HS \\
\hline Inferior perifoveal macula & 231.00 & 292.00 & 253.60 & 17.80 & 230.00 & 269.00 & 258.10 & 10.56 & 1.38 & 1.38 & NS \\
\hline Nasal perifoveal macula & 215.00 & 304.00 & 269.22 & 21.27 & 249.00 & 307.00 & 292.22 & 14.59 & 5.64 & 5.64 & HS \\
\hline Foveal center & 166.00 & 290.00 & 213.90 & 23.21 & 192.00 & 289.00 & 237.73 & 24.74 & 4.44 & 4.44 & HS \\
\hline
\end{tabular}

*: Student $t$-test.

This table present the predictor of changes detected on macular thickness according OCP group vs normal group. Most of layers of Macular are highly significant with a $p$-value $<0.01$. But inferior outer macular layer is non-significant with $p$-value $<0.05$.

Table (3): Comparison between OCP group and normal group regarding macular volume.

\begin{tabular}{|c|c|c|c|c|c|c|c|c|c|c|c|}
\hline \multirow{2}{*}{$\mathrm{OP}$} & \multicolumn{4}{|c|}{ OCP group $(\mathrm{N}=40)$} & \multicolumn{4}{|c|}{ Normal group $(\mathrm{N}=40)$} & \multirow{2}{*}{$t^{*}$} & \multirow{2}{*}{$\begin{array}{c}p- \\
\text { value }\end{array}$} & \multirow{2}{*}{ Sig. } \\
\hline & Min. & Max. & Mean & SD & Min. & Max. & Mean & SD & & & \\
\hline Average macular volume & 6.95 & 7.97 & 7.38 & .36 & 6.78 & 9.90 & 7.84 & .72 & 3.60 & 0.001 & $\mathrm{HS}$ \\
\hline
\end{tabular}

This table present the predictor of changes detected on macular volume according OCP group
Vs. normal group. Which is highly significant with a $p$-value $<0.001$.

Table (4): Comparison between OCP group and normal group regarding RNFL.

\begin{tabular}{|c|c|c|c|c|c|c|c|c|c|c|c|}
\hline & \multicolumn{4}{|c|}{ OCP group $(\mathrm{N}=40)$} & \multicolumn{4}{|c|}{ Normal group $(\mathrm{N}=40)$} & \multirow{2}{*}{$t^{*}$} & \multirow{2}{*}{$\begin{array}{c}p- \\
\text { value }\end{array}$} & \multirow{2}{*}{ Sig. } \\
\hline & Min. & Max. & Mean & SD & Min. & Max. & Mean & $\mathrm{SD}$ & & & \\
\hline Temporal & 55.00 & 94.00 & 77.77 & 9.49 & 62.00 & 85.00 & 75.20 & 6.98 & 1.38 & 0.171 & NS \\
\hline Nasal & 62.00 & 112.00 & 85.37 & 13.88 & 60.00 & 79.00 & 67.72 & 5.65 & 7.45 & $<0.001$ & HS \\
\hline Superior & 104.00 & 162.00 & 121.85 & 15.16 & 99.00 & 145.00 & 128.40 & 12.15 & 2.13 & 0.036 & $\mathrm{~S}$ \\
\hline Inferior & 79.00 & 161.00 & 127.83 & 18.69 & 99.00 & 124.00 & 110.62 & 7.32 & 5.42 & $<0.001$ & HS \\
\hline Average & 79.00 & 122.00 & 102.88 & 9.73 & 80.00 & 105.00 & 96.50 & 6.27 & 3.48 & 0.001 & HS \\
\hline
\end{tabular}

*: Student $t$-test.

Table (5): Comparison between OCP group and normal group regarding choroid thickness.

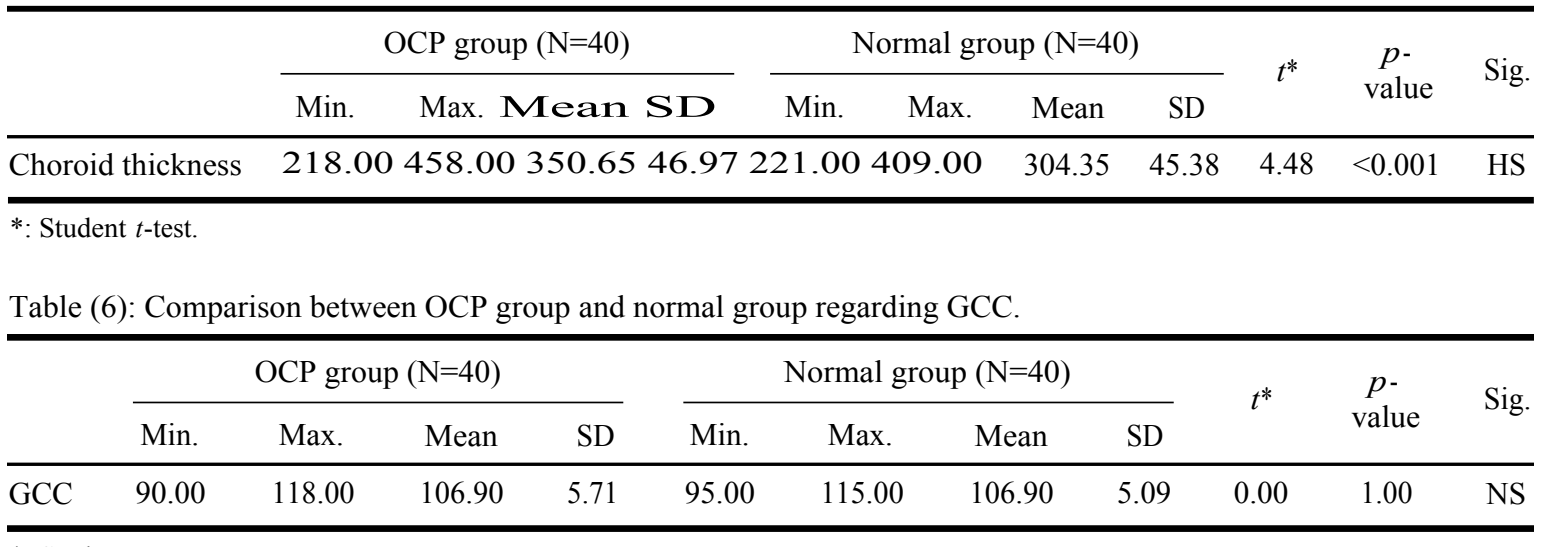

*: Student $t$-test. 
Table (4) present the predictor of changes detected on retinal nerve fiber layer according OCP group vs normal group. Nasal, inferior, and average RNFL are highly significant with a $p$-value $<0.01$, superior RNFL is significant wit $p$-value $<0.05$. But, temporal RNFL zone is non-significant with $p$-value $<0.05$.

Table (5) present the predictor of changes detected choroid thickness according OCP group vs normal group. which is highly significant with a $p$-value $<0.001$.

Table (6) present the predictor of changes detected Ganglion Cell Complex (GCC) according OCP group Vs. normal group which is no significant with a $p$-value $>0.05$.

\section{Discussion}

In partial agreement of our results, Yusuf et al., [19]. Studied 24 healthy women taking monophasic OCP $(3 \mathrm{mg}$ drospirenone and $0.03 \mathrm{mg}$ ethinylestradiol) for contraception only for at least one year in comparison to 24 healthy women who were not using an OCP. Supported that all measurements of posterior ocular segments, except foveal center thickness, were considerably slimmer in the OCT group versus to the control group. All macular volume variable values were considerably lower in the OCP group versus the control group. It reports that, although macular slimming May be a result of aging or degeneration. It may be with no clinical significance.

On the other hand, Malek et al., [6] suggest that retinal vascular diseases such as occlusion of the central retinal artery, intraocular hemorrhage, and more rarely macular edema have been reported retrospectively as an adverse ocular reaction to oral contraceptives (OCs).

The study shows that, the average of the RNFL, nasal (N), and inferior (I) parts of the RNFL were considerably thicker in the OCP group versus the control group with ( $p=<0.001, p=<0.001, p=0.001$, respectively) while superior (S) part slightly thinner in OCP study group than control group.

In contrary to our results, Yusuf et al., [19] found that the average RNFL thickness, particularly the NI and TI parts, was significantly decreased in women using OCP. However, this decrease was not clinically significant. Suggesting that the use of OCP produce effect mimics age-related changes. On the other hand, progesterone may counteract vasodilatation effect of estrogen by reducing endothelial nitric oxide production. Drospirenone, which was the progesterone derivate used in the study, has anti-mineralocorticoid and anti-edema effect.

According to previous study we observed that 6 eyes of 40 eyes had examined in our study group taking oral contraceptive pills. Show RNFL thinning specially in NI, and TS parts which counts $15 \%$ of total cases have thinning of Retinal nerve fiber layer.

The thickening in RNFL may be supported by Malek et al., [6] who suggest ed that oral contraceptives appear to increase the incidence of benign intracranial hypertension, manifested by headaches, papillary edema endangering the optic nerve, and the absence of visible anomalies on the imaging.

Though, the role of female sex hormones in IIH is not clear, but estrogen is found to be associated with a prothrombotic state and, subject to the theory of micro-thrombotic obstruction of arachnoid villi. It may also decrease tight junctional resistance and remodeling of occluding which directly affects paracellular permeability. Paracellular permeability is important in the exchange of water between the CSF and brain via the ependymal and pial lining.

Yusuf et al., [19] support the fact that oral contraceptive pills effects have been shown to change depending on the type of progesterone used. So, in comparison to our study results. Gestodene (third-generation progestin) is a synthetic progestogen used in our study. It has a weak androgenic activity, weak glucocorticoid activity, weak antimineralocorticoid activity, and weak anti-edema effect.

As regard to Choroid Thickness (CT), it is significantly thicker in OCP study group than control group. This result is contraversed to other studies.

Yusuf et al., [19] suggest that there was no statistically significant difference between two groups in terms of CT. Explaining that using a combined oral contraceptive could be the cause of unaffected choroid thickness, because there was no unopposed estrogen action on the women using OCP. Choroid thickness was significantly increased in women with Polycystic Ovary Syndrome (PCOS) compared to healthy women this was due to increased estrogen-dependent vasodilatory action in the ophthalmic artery, related to high estrogen levels or unopposed estrogen, in the women with PCOS. 
Based on our measurement on the Ganglion Cell Complex (GCC). In our study, there was no statistically significant difference between two groups in terms of GCC.

This result is supported by that estrogen has neuroprotective effect, by protecting neurons via a variety of mechanisms; e.g., by interfering with cellular death signaling cascades, altering neurotransmitters levels, polish up the free radicals, as well as promoting synaptic plasticity.

Dawn [20] stated that there are many types of progestins, and each has a different profile in terms of progestational, estrogenic, and androgenic activity effects. The result of these effects is dependent on the combination of the type and level of progestin and the level of estrogen. Progestins with higher androgenic activity may increase the chances of androgen-related side effects. On other hand, a higher number of micrograms of estrogen (ethinyl estradiol) lead to more potent estrogenic effects. A higher amount of estrogenic activity helps to decrease androgen-related side effects. However, progestins tend to counter some of the estrogenic effects of ethinyl estradiol.

\section{Limitations:}

Of this study include, the long-term consequences of these changes which need a closer observation. Very short duration of the study.

Obesity, alcohol intake, and smoking are a great risk factors that may influence the side effects of oral contraceptive pills on eye. Which are not focused on this study.

There are plenty of ocular changes occur due to receiving oral contraceptive pills for long period not only posterior segment needed to be assessed. So, female who are taking OCP are recommended to have annual ocular examination and follow-up.

\section{Conclusion:}

In our study, we found that women who had received combined oral contraceptive pills more than one year have changes in posterior ocular segment.

\section{References}

1- FUCHSJAGER-MAYRL G., NEPP J., et al.: Identification of estrogen and progesterone receptor mRNA expression in the conjunctiva of premenopausal women. Invest. Ophthalmol. Vis. Sci., 43 (9): 2841-44, 2002.

2- ADEYEMI A.S., ADEKANLE D.A. and KOMOLAFE J.O.: Pattern of contraceptives choice among the married women attending the family planning clinic of a tertiary health institution. Niger. J. Med., 2008; 17: 67-70. Gold- haber SZ. Risk factors for venous thromboembolism. J. Am. Coll. Cardiol., 56 (1): 1-7, 2010.

3- RADNOT M. and FOLLMANN P.: Ocular side-effects of oral contraceptives. Ann. Clin. Res., 5: 197-204, 1973.

4- STOCCO B., FUMAGALLI H.F., FRANCESCHINI S.A., MARTINEZ E.Z., MARZOCCHI MACHADO C.M., De SÁ M.F. and TOLOI M.R.: Comparative study of the effects of combined oral contraceptives in hemostatic variables: An observational preliminary study. Medicine (Baltimore), 94 (4): e385, 2015.

5- LEFF S.P.: Side-effect of oral contraceptives: Occlusion of branch artery of the retina. Bull Sinai Hosp. Detroit., 24 (4): 227-9, 1976.

6- MALEK N. and LEBUISSON D.A.: Adverse ocular reactions to oral contraceptive use. Contracep. tFertil Sex (Paris), 20 (4): 441-4, 1992

7- ANTHONY KWONG O.D.: Eye health and oral contraceptives: A woman's risks, Retrieved from http:// healthylivingmadesimple.com/eve-health-and-oral-contraceptivesa-womans-risks, 2014.

8- SONALKAR S., SCHREIBER C.A. and BARNHART K.T.: Contraception. De Groot L.J., Beck-Peccoz P., Chrousos G., Dungan K., Grossman A., Hershman J.M., Koch C., McLachlan R., New M., Rebar R., Singer F., Vinik A., Weickert M.O. Endocrinology of Female Reproduction South Dartmouth (MA), USA. MDText.com, Inc., 2000-2014 Nov 11.

9- GUPTA P.D., KAID JOHAR, NAGPAL K. and VASAVADA A.R.: Sex hormone receptors in the human eye. Surv. Ophthalmol., 50 (3): 274-84, 2005.

10- SHAN L.: Using-birth-control-pills-may-increase-womensglaucoma-risk, Retrieved from https://www.npr.org/ sections/health-shots/2013/11/18/ 245959355/ using-birthcontrol-pills-may-increase-womens-glaucoma-risk, 2013.

11- MOSCHOS M.M. and NITODA E.: The impact of combined oral contraceptives on ocular tissues: A review of ocular effects. Int. J. Ophthalmol., 10 (10): 1604-10, 2017.

12- MEENDERING J.R., TORGRIMSON B.N., MILLER N.P., et al.: Ethinyl estradiol-to-desogestrel ratio impacts endothelial function in young women. Contraception, 79: 41-9, 2009.

13- SCHNAPER H.W., McGUIRE J., RUNYAN C. and HUBCHAK S.C.: Sex steroids and the endothelium. Curr. Med. Chem., 7: 519-31, 2000.

14- MEENDERING J.R., TORGRIMSON B.N., MILLER N.P., et al.: Ethinyl estradiol-to-desogestrel ratio impacts endothelial function in young women. Contraception, 79: 41-9, 2009.

15- MINER J.A., MARTINI E.R., SMITH M.M., et al.: Shortterm oral progesterone administration antagonizes the effect of transdermal estradiol on endothelium-dependent vasodilation in young healthy women. Am. J. Physiol. Heart Circ. Physiol., 301: 1716-22, 2011.

16- WIEGRATZ I. and KUHL H.: Metabolic and clinical effects of progestogens. Eur. J. Contracept. Reprod Health Care, 11 (3): 153-61, 2006.

17-AREF A.A. and BUDENZ D.L.: Spectral domain optical coherence tomography in the diagnosis and management 
of glaucoma. Ophthalmic. Surg. Lasers Imaging, 41: S 1527, 2010.

18- MURTHY R.K., HAJI S., SAMBHAV K., GROVER S. and CHALAM K.V.: Clinical applications of spectral domain optical coherence tomography in retinal diseases. Biomed. J., 39: 107-20, 2016.

19- YUSUF M., GOKHAN A., MUSTAFA A., ERDEM S.,
AHTER T.T., ILKNUR Ç.M., FATMA O. and VESILE S.: The Effect of Oral Contraceptive Pills on the Macula, the Retinal Nerve Fiber Layer, and Choroidal Thickness. Med. Sci. Monit., 23: 5657-61, 2017.

20- DAWN S.: Types of Combination Birth Control Pills Differences between Monophasic, Biphasic, and Triphasic Pills., 27 (1): 3-12, 2018.

\section{تقييم طبقة الألياف العصبية للشبكية البقعة وسمك المشيمة عند النساء اللآئى يستخلدمن حبوب منع الحمل عن طريق الفم}

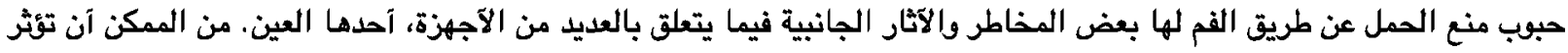

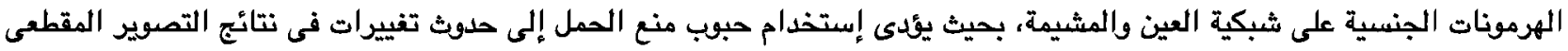

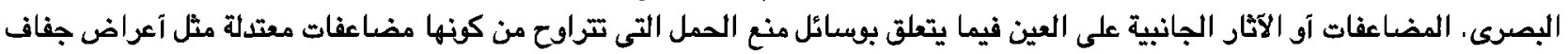



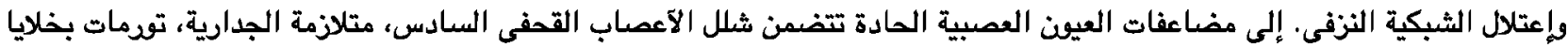

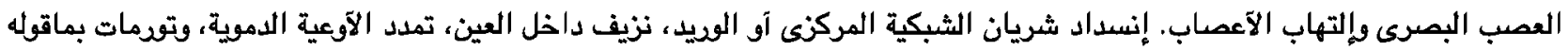
العين وإعتلال عصبى بصرى حاد.

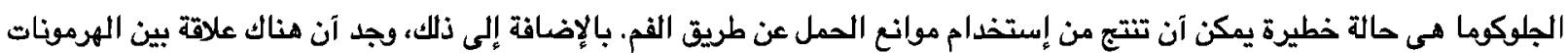

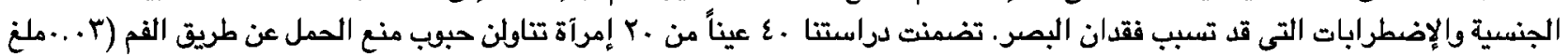

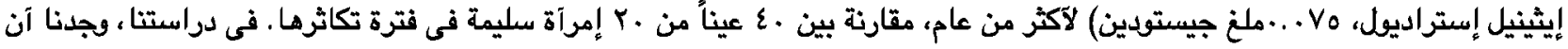



